Article

\title{
Synthesis of Sulfonic Acid-Functionalized Zirconium Poly(Styrene-Phenylvinyl-Phosphonate)-Phosphate for Heterogeneous Epoxidation of Soybean Oil
}

\author{
Xiaochuan Zou ${ }^{1} \oplus$, Xuyuan Nie ${ }^{1}$, Zhiwen Tan ${ }^{2}$, Kaiyun Shi ${ }^{1}{ }^{\oplus}$, Cun Wang ${ }^{1}$, Yue Wang ${ }^{1}$ and \\ Xin Zhao $1,3,4,5, *$ (D) \\ 1 Department of Biological and Chemical Engineering, Chongqing University of Education, Nan'an 400067, \\ China \\ 2 College of Chemistry, Chongqing Normal University, Chongqing 401331, China \\ 3 Chongqing Collaborative Innovation Center of Functional Food, Chongqing University of Education, \\ Chongqing 400067, China \\ 4 Chongqing Engineering Technology Research Center for Functional Food, Chongqing University of \\ Education, Chongqing 400067, China \\ 5 Chongqing Engineering Laboratory of Functional Food, Chongqing University of Education, \\ Chongqing 400067, China \\ * Correspondence: zhaoxin@cque.edu.cn; Tel.: +86-23-8630-7018; Fax: +86-23-6163-8000
}

Received: 5 August 2019; Accepted: 21 August 2019; Published: 24 August 2019

\begin{abstract}
In this paper, a solid acid catalyst (ZPS-PVPA-SO ${ }_{3} \mathrm{H}$ ) was prepared by anchoring thiol group on zirconium poly(styrene-phenylvinyl-phosphonate)-phosphate (ZPS-PVPA), followed by oxidation of thiol groups to obtain sulfonic acid groups. The solid acid catalyst was characterized by XPS, X-ray, EDS, SEM, and TG-DSC. The successful preparation of sulfonic acid-functionalized ZPS-PVPA was confirmed. Subsequently, the catalytic performance of ZPS-PVPA-SO ${ }_{3} \mathrm{H}$ was investigated in the epoxidation of soybean oil. The results demonstrated that $\mathrm{ZPS}-\mathrm{PVPA}-\mathrm{SO}_{3} \mathrm{H}$ can effectively catalyze epoxidation of soybean oil with TBHP as an oxidant. Moreover, there was no significant decrease in catalytic activity after 5 repeated uses of the ZPS-PVPA-SO ${ }_{3} \mathrm{H}$. Interestingly, the ZPS-PVPA-SO ${ }_{3} \mathrm{H}$ was kept in $2 \mathrm{~mol} / \mathrm{L}$ of $\mathrm{HCl}$ overnight after the end of the seventh reaction, and the catalytic activity was gradually restored during the eighth to tenth cycles.
\end{abstract}

Keywords: solid acid; zirconium poly(styrene-phenylvinyl-phosphonate)-phosphate; heterogeneous catalysis; epoxidation of soybean oil

\section{Introduction}

The epoxidation of vegetable oil has attracted much attention because the obtainable epoxides are useful for producing a wide range of products, such as plasticizers and stabilizers of polyvinyl chloride (PVC) [1]. Currently, these products are mainly prepared by using a peracid [2,3] in the presence of protonic acid catalysts, such as sulfuric acid [4]. However, separation and reuse of such protonic acid catalysts are still problematic for the reaction mixture [3]. In addition, protonic acid catalysts can also pollute the environment, posing a hazard and causing more by-products. Therefore, a new type of effective catalyst is urgently needed for epoxidation of soybean oil.

In the last few decades, a large number of functional catalytic materials containing $-\mathrm{SO}_{3} \mathrm{H}$ groups have been reported [5-8]. The $-\mathrm{SO}_{3} \mathrm{H}$-based functionalized catalytic materials can not only replace liquid acids to catalyze various reactions and reduce wastewater, but also have significant shape-selective catalysis. Their nature also increases the selectivity of the reaction and reduces the by-products of the reaction. Until now, the main choices of catalytic materials have been zeolites, silica, 
$\alpha$-zirconium phosphate, and metal oxides [9-11]. However, $-\mathrm{SO}_{3} \mathrm{H}$-based-organic polymer-inorganic hydrogen zirconium phosphate catalytic materials applied to epoxidation of soybean oil have not been reported.

Organic polymer-inorganic hydrogen zirconium phosphate hybrid materials have been central to many areas of chemistry because they have the properties of organic and inorganic ingredients [12,13]. It is mainly because these materials have different hydrophilic or hydrophobic properties (regulating the ratio of organic polystyrene segments to inorganic zirconium hydrogen phosphate components), specific surface areas and holes of different sizes (regulating the ratio of organic polymers), and nanolayer structure. In addition, the surface of the organic polymer-inorganic hydrogen zirconium phosphate also contains a large amount of benzene rings and $-\mathrm{OH}$, which can perform different functionalization reactions.

In our previous works [14,15], the organic polymer-inorganic hydrogen zirconium phosphate, which were frequently functionalized on the benzene ring in the organic component, has been demonstrated to be a versatile support to immobilize the homogeneous chiral MnIII (salen) complex. The corresponding works demonstrated that ZPS-PVPA-based catalyst can effectively catalyze $\alpha$-methylstyrene and indene, especially $\alpha$-methylstyrene; the conversion and enantioselectivity are $99 \%$. Moreover, the ZPS-PVPA- $\mathrm{SO}_{3} \mathrm{H}$ catalysts, which were synthesized by the reaction of a chloromethyl group on the benzene ring in ZPS-PVPA and an additional sulfonic acid group, can effectively promote the catalytic epoxidation of soybean oil [16]. However, one of the biggest problems is the use of carcinogenic chloromethyl ether during the functionalization of hybrid materials. In addition, the process was complicated and had a low degree of functionalization.

Hence, the need to better understand these new functionalized hybrid materials and find a more stable, efficient, simple, and reusable solid acid catalyst encourage us to make further efforts. Considering the rich -OH on the surface of ZPS-PVPA and avoiding the use of carcinogenic chloromethyl ether, we extend our attempts to prepare $\mathrm{ZPS}-\mathrm{PVPA}-\mathrm{SO}_{3} \mathrm{H}$ by the condensation reaction between the -OH on the surface of ZPS-PVPA and the alkoxyl- or chloro-silane groups of organic molecules. Then, the catalytic performance of ZPS-PVPA- $\mathrm{SO}_{3} \mathrm{H}$ was investigated in the epoxidation of soybean oil. The results demonstrated that the $\mathrm{ZPS}-\mathrm{PVPA}-\mathrm{SO}_{3} \mathrm{H}$ can effectively catalyze epoxidation of soybean oil with TBHP as an oxidant. Moreover, the ZPS-PVPA-SO ${ }_{3} \mathrm{H}$ could be easily separated from the reaction system and recycled up to at least five times without significant loss of catalytic activity. Interestingly, ZPS-PVPA-SO ${ }_{3} \mathrm{H}$ was kept in $2 \mathrm{~mol} / \mathrm{L}$ of $\mathrm{HCl}$ for overnight and after the end of the seventh reaction, the catalytic activity was gradually restored during the eighth to tenth cycles.

\section{Results and Discussion}

\subsection{The Acidity of the Samples of Solid Particles}

The calculated acidities of ZPS-PVPA, ZPS-PVPA-SH and ZPS-PVPA-SO ${ }_{3} \mathrm{H}$ are presented in Table 1 . The acidity of ZPS-PVPA microcrystals is mainly caused by the -OH within ZPS-PVPA layers [17]. When -SH group terminated chains were grafted onto ZPS-PVPA, the acidity of ZPS-PVPA-SH decreased, indicating the successful condensation reaction between the $-\mathrm{OH}$ of individual ZPS-PVPA and the -Si(OMe $)_{3}$ groups of MPTMS. After the ZPS-PVPA-SH nanosheets were oxidized to ZPS-PVPA-SO ${ }_{3} \mathrm{H}$, the acidity was sharply enhanced to $418.9 \mu \mathrm{mol} / \mathrm{g}$ from $0.12 \mu \mathrm{mol} / \mathrm{g}$, which in turn confirms the formation of $-\mathrm{SO}_{3} \mathrm{H}$ groups.

Table 1. Acidity of the samples of solid particles.

\begin{tabular}{ccc}
\hline Number & Sample & Acidity $(\mu \mathrm{mol} / \mathrm{g})$ \\
\hline 1 & ZPS-PVPA & 7.2 \\
2 & ZPS-PVPA-SH & 0.12 \\
3 & ZPS-PVPA-SO ${ }_{3} \mathrm{H}$ & 418.9 \\
\hline
\end{tabular}




\subsection{X-Ray Photoelectron Spectroscopy}

The XPS results for ZPS-PVPA, ZPS-PVPA-SH and ZPS-PVPA-SO ${ }_{3} \mathrm{H}$ are summarized in Figure 1. The appearance of $S$ and $\mathrm{Si}$ elements on the surface of ZPS-PVPA-SH confirmed the successful grafting of MPTMS on the ZPS-PVPA. Moreover, the XPS spectra of P 2p for ZPS-PVPA, ZPS-PVPA-SH, and ZPS-PVPA-SO ${ }_{3} \mathrm{H}$ were recorded and are shown in Figure 2. Compared to ZPS-PVPA, the binding energy of the P 2p peak of ZPS-PVPA-SH increased from 134.04 to $134.08 \mathrm{eV}$ after functional reaction. This result is consistent with the formation of $\mathrm{P}-\mathrm{O}-\mathrm{Si}$ bonds between the $-\mathrm{OH}$ on the surface of ZPS-PVPA and the $-\mathrm{Si}(\mathrm{OMe})_{3}$ groups of MTPMS. In addition, the binding energy values of $\mathrm{P} 2 \mathrm{p}$ for ZPS-PVPA-SH and ZPS-PVPA-SO ${ }_{3} \mathrm{H}$ are both $134.08 \mathrm{eV}$, which indicates that the oxidation of ZPS-PVPA-SH has no effect on the chemical bonds between the ZPS-PVPA and the grafted chains, ensuring that the chains remain grafted on the ZPS-PVPA surface after oxidation reaction. To further confirm that the $-\mathrm{SH}$ groups were oxidized into $-\mathrm{SO}_{3} \mathrm{H}$ groups under the influence of $\mathrm{H}_{2} \mathrm{O}_{2}$ and $\mathrm{HCl}$, the XPS spectra of $S 2 p 3 / 2$ were carried out, and the results are shown in Figure 3. A higher binding energy of $\mathrm{S} 2 \mathrm{p} 3 / 2(168.1 \mathrm{eV}$ Vs $163.4 \mathrm{eV})$ was obtained after the oxidation, which in turns proves that the ZPS-PVPA-SH were successfully oxidized into ZPS-PVPA- $\mathrm{SO}_{3} \mathrm{H}$.

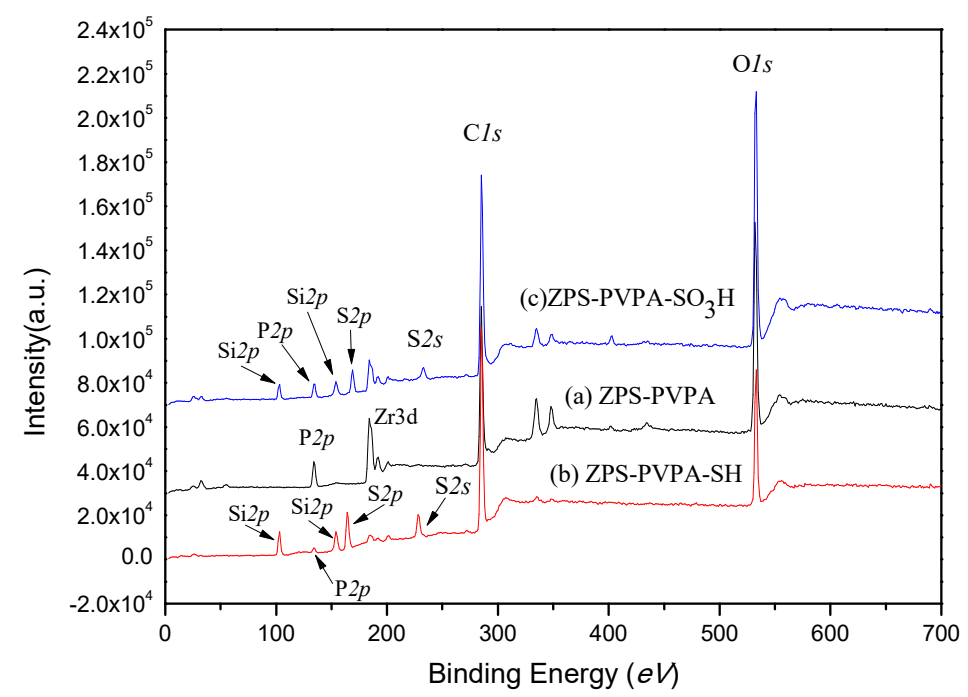

Figure 1. XPS survey scan of (a) ZPS-PVPA, (b) ZPS-PVPA-SH, and (c) ZPS-PVPA-SO ${ }_{3} \mathrm{H}$.

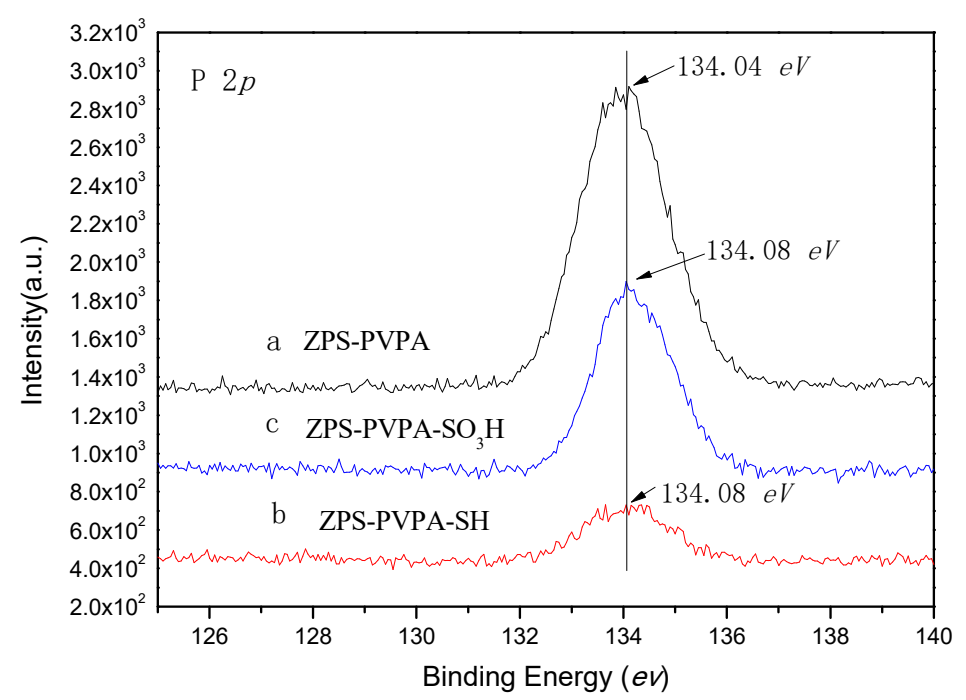

Figure 2. XPS of P 2p peaks of (a) ZPS-PVPA, (b) ZPS-PVPA-SH, and (c) ZPS-PVPA-SO ${ }_{3} \mathrm{H}$. 


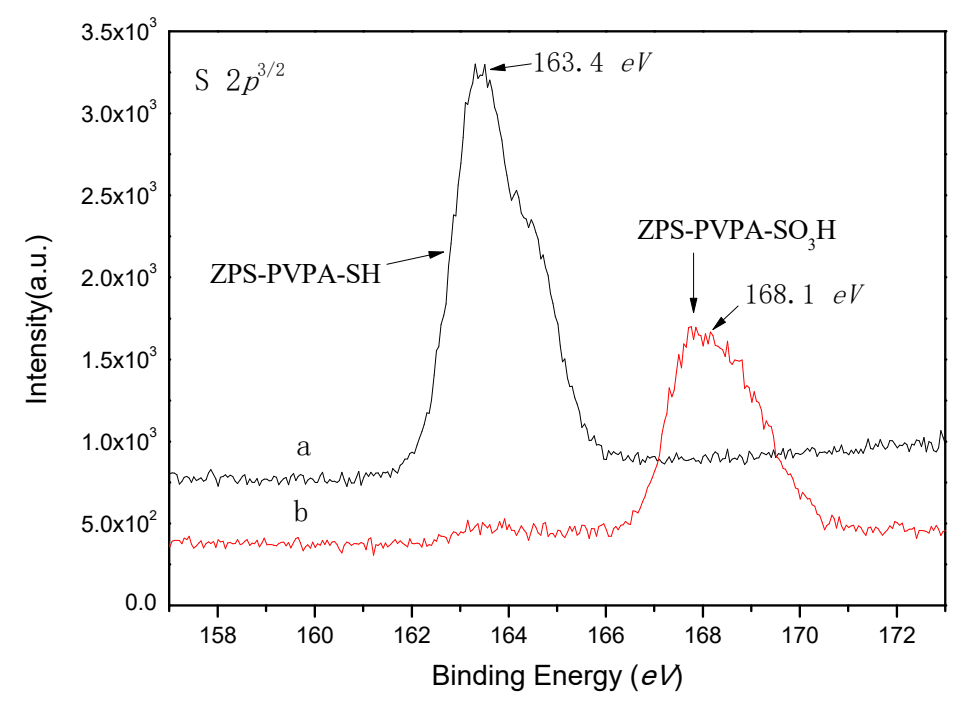

Figure 3. XPS of S 2p3/2 peaks of (a) ZPS-PVPA-SH and (b) ZPS-PVPA-SO ${ }_{3} \mathrm{H}$.

\subsection{XRD Analysis}

Figure 4 presents the XRD patterns of ZPS-PVPA, ZPS-PVPA-SH, and ZPS-PVPA-SO ${ }_{3} \mathrm{H}$. It can be seen from the $X R D$ spectrum that there has no obvious peak, indicating that the surface morphologies of the ZPS-PVPA, ZPS-PVPA-SH, and ZPS-PVPA-SO ${ }_{3} \mathrm{H}$ are amorphous. The broad peak at $2 \theta=15.0-30^{\circ}$ is mainly attributed to the amorphous silica. Furthermore, the fine diffraction peaks in ZPS-PVPA, ZPS-PVPA-SH and ZPS-PVPA-SO ${ }_{3} \mathrm{H}$ are attributed to the special structure of the organic-inorganic hybrid zirconium phosphate. Theoretically, each of the composite zirconium salt particles is composed of a plurality of layered composite zirconium salt crystallites, and the arrangement of the crystal planes of the respective zirconium salt crystal grains is random. Therefore, the results are very likely to present a form of "short-range order, long-range disorder".

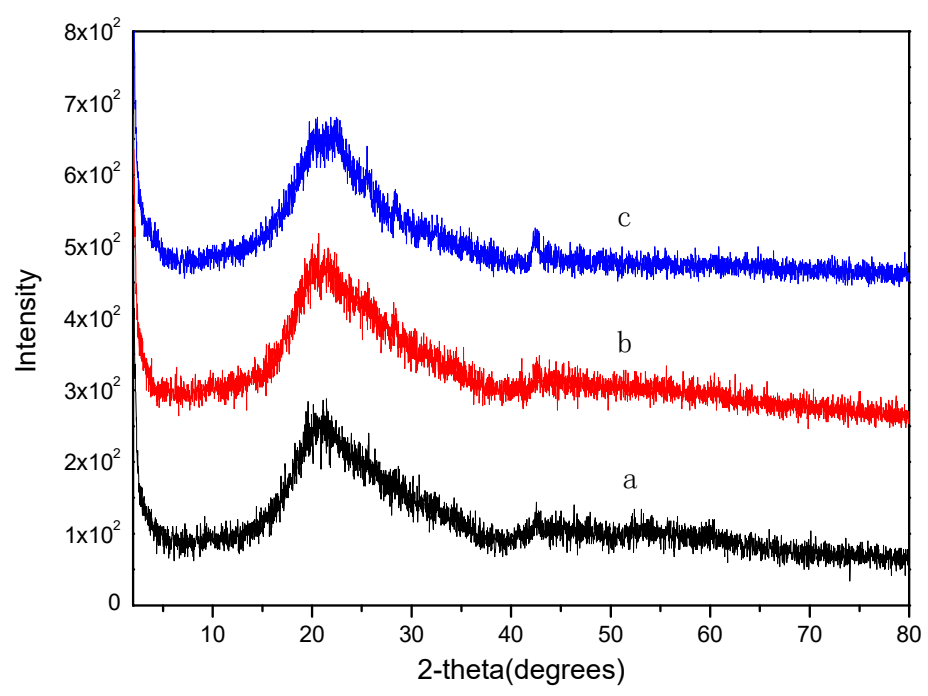

Figure 4. XRD patterns of solid particles: (a) ZPS-PVPA, (b) ZPS-PVPA-SH, and (c) ZPS-PVPA-SO 3 H. 


\subsection{Microscopic Analysis}

SEM images provide direct information on the microstructure and morphology of ZPS-PVPA and ZPS-PVPA-SO ${ }_{3} \mathrm{H}$, as shown in Figure 5. In this context, Figure 5a indicates that the ZPS-PVPA was amorphous and loose, and various cavities, holes, and pores were present in every particle in ZPS-PVPA. These pores and cavities of varying sizes are caused by the disorderly arrangement of organic polymer and layered crystallites of inorganic zirconium phosphate. Figure $5 b$ shows that the structure of ZPS-PVPA-SO${ }_{3} \mathrm{H}$ is still amorphous, loose, and porous. However, compared to ZPS-PVPA, the channels and pores of ZPS-PVPA- $\mathrm{SO}_{3} \mathrm{H}$ are more closely packed, which is mainly due to the insertion of the $-\mathrm{SO}_{3} \mathrm{H}$ group and results in an increase in the interaction between the particles during the sulfonation process. The EDS surveys of ZPS-PVPA and ZPS-PVPA-SO ${ }_{3} \mathrm{H}$ are presented in Figure $5 \mathrm{c}, \mathrm{d}$. The additional peaks of $\mathrm{S}$ and $\mathrm{Si}$ elements in ZPS-PVPA-SO $\mathrm{S}_{3} \mathrm{H}$ further confirm the successful grafting and oxidation of $-\mathrm{SH}$ into $-\mathrm{SO}_{3} \mathrm{H}$ group. Furthermore, we carried out element mapping of $\mathrm{ZPS}-\mathrm{PVPA}-\mathrm{SO}_{3} \mathrm{H}$ (Figure $6 \mathrm{a}-\mathrm{f}$ ). Considerable quantities of $\mathrm{Si}$ and $\mathrm{S}$ were detected, along with $\mathrm{Zr}, \mathrm{P}, \mathrm{C}$, and $\mathrm{O}$, implying the successful grafting of MPTMS on the ZPS-PVPA and oxidation of $-\mathrm{SH}$ into $-\mathrm{SO}_{3} \mathrm{H}$ group.
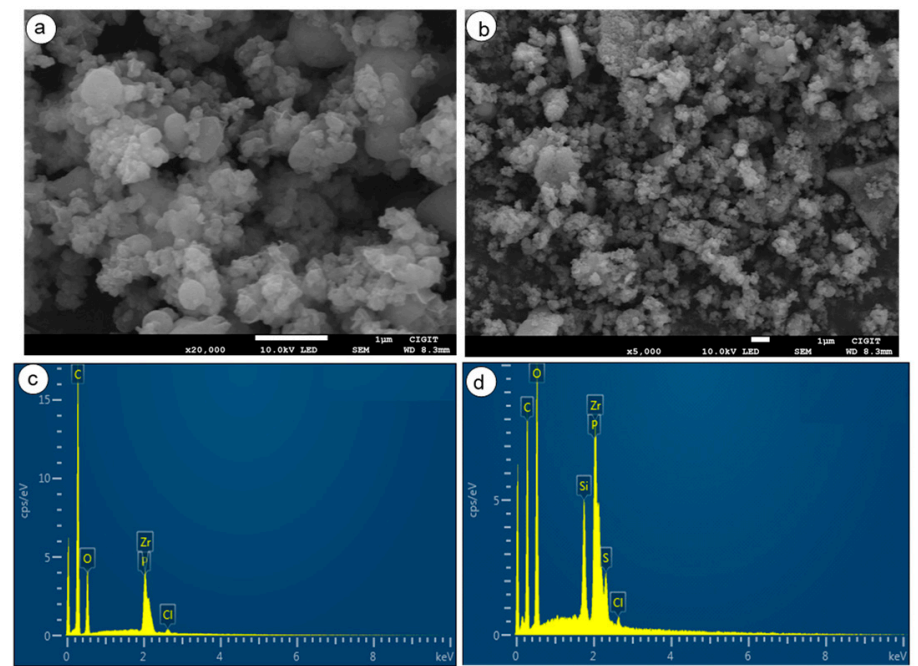

Figure 5. SEM photograph of (a) ZPS-PVPA, (b) ZPS-PVPA-SO $\mathrm{S}_{3} \mathrm{H}$, and the measured EDS images of (c) ZPS-PVPA, (d) ZPS-PVPA-SO 3 H. 
C K $\alpha 1 \_2$
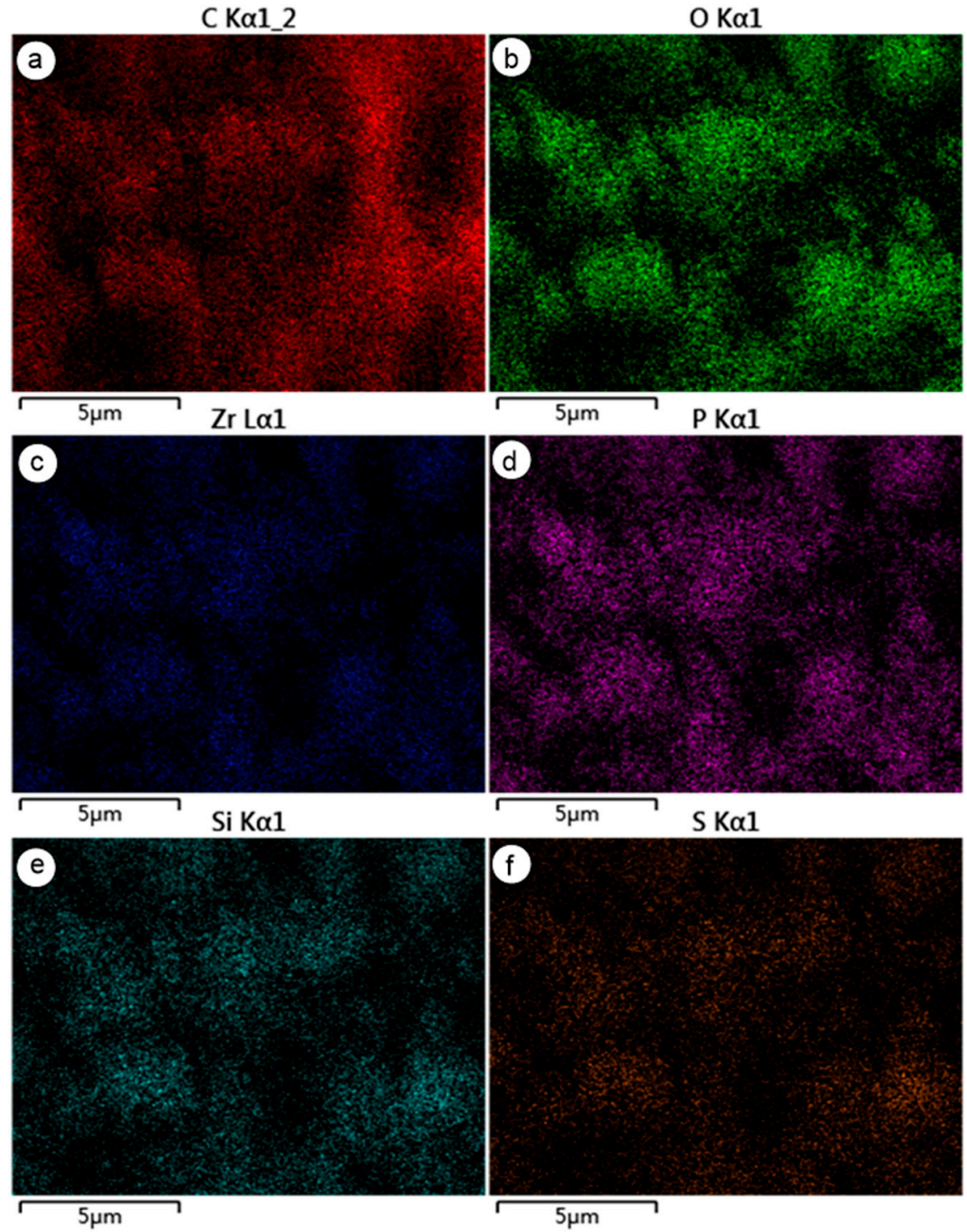

Figure 6. EDX elementary mapping of (a) $\mathrm{C},(\mathbf{b}) \mathrm{O},(\mathbf{c}) \mathrm{Zr},(\mathbf{d}) \mathrm{P},(\mathbf{e}) \mathrm{Si}$, and (f) $\mathrm{S}$ in the catalyst ZPS-PVPA-SO 3 H.

\subsection{Thermal Gravimetric Analysis}

The thermal stability of the catalyst ZPS-PVPA- $\mathrm{SO}_{3} \mathrm{H}$ was determined by thermogravimetric analysis (TG) and differential scanning calorimetry (DSC) (Figure 7). The weight loss of the catalyst ZPS-PVPA- $\mathrm{SO}_{3} \mathrm{H}$ is divided into four processes. It can be seen from the TG-DSC curve that the first process is the temperature increase from room temperature to $120^{\circ} \mathrm{C}$, where weight loss occurs at $8.7 \%$, and the corresponding endothermic peak appears at $63.2^{\circ} \mathrm{C}$ in the DSC curve, which is attributed to the desorption of surface adsorption water and crystal water in the solid catalyst. The second process was the temperature increase from 155 to $222^{\circ} \mathrm{C}$ where a weight loss of $2.9 \%$ occurs, which is still due to the removal of residual solid water from the catalyst. The third stage is the temperature increase from 222 to $706^{\circ} \mathrm{C}$, resulting in mass loss of $43.8 \%$ and two obvious exothermic peaks occurring simultaneously at 426.3 and $530.4^{\circ} \mathrm{C}$, which is mainly attributed to the decomposition of organic groups and burning of species carbon in the catalyst ZPS-PVPA- $\mathrm{SO}_{3} \mathrm{H}$. Furthermore, the organophosphine is oxidized to pentavalent phosphorus and $\mathrm{Zr}\left(\mathrm{HPO}_{4}\right)_{2}$ is formed at this stage, and the organic group is almost completely destroyed at $706{ }^{\circ} \mathrm{C}$. The fourth stage is the temperature increase from 706 to $800{ }^{\circ} \mathrm{C}$, accompanied by a strong endothermic peak at $667.2^{\circ} \mathrm{C}$. After heating to $800{ }^{\circ} \mathrm{C}$, white residual powder is obtained, which is mainly due to the dehydration of $\mathrm{Zr}\left(\mathrm{HPO}_{4}\right)_{2}$ to form zirconium pyrophosphate 
$\mathrm{ZrP}_{2} \mathrm{O}_{7}$. The quality loss is not obvious. From the above thermogravimetric analysis, it can be stated that the thermal stability of the catalyst ZPS-PVPA- $\mathrm{SO}_{3} \mathrm{H}$ is reached at about $230^{\circ} \mathrm{C}$.

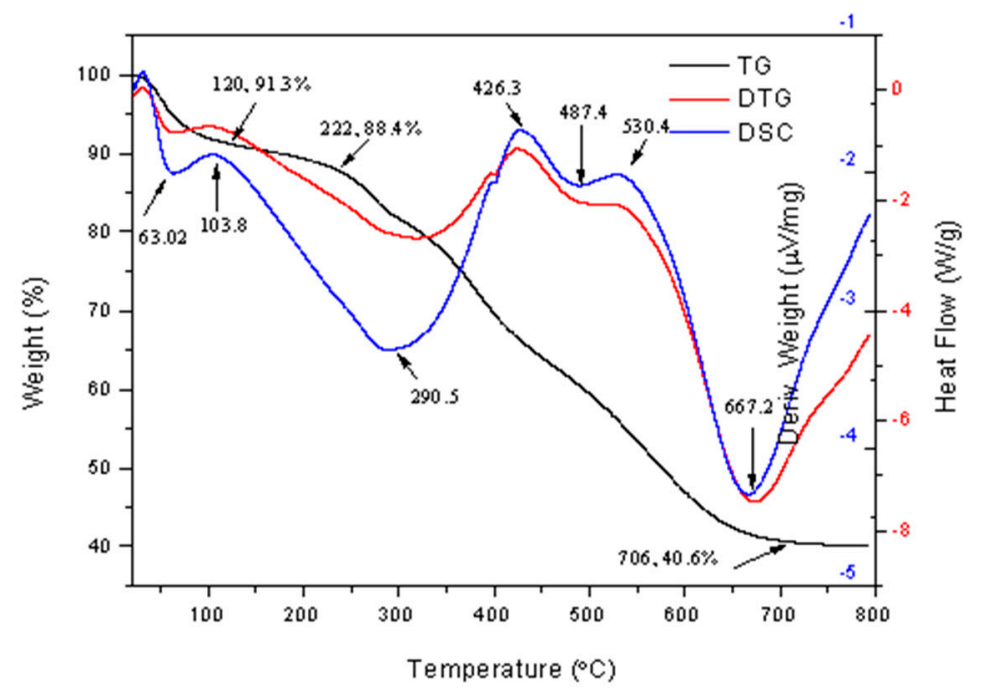

Figure 7. The TG-DSC curves of the ZPS-PVPA-SO $\mathrm{SO}_{3} \mathrm{H}$

\subsection{Catalytic Reaction}

Table 2 summarizes the results of the epoxidation of soybean oil over the catalyst ZPS-PVPA-SO 3 H. All reactions proceeded smoothly, and these results are significantly better than those catalyzed by the Schiff base molybdenum (VI) [18] and the $\mathrm{Cu}$ (salen) complex [19] under optimal catalytic conditions. The excellent catalytic activity is mainly attributed to the special structure of ZPS-PVPA- $\mathrm{SO}_{3} \mathrm{H}$, which facilitate self-assembly into a plurality of micro-reactors. Thus, the particles of one catalyst are randomly stacked by hundreds of solid catalyst crystallites. The catalytic active centers are typically located in the channels or inner or outer layers of ZPS-PVPA. When the catalyst is in the TBHP oxidation system, the layers of ZPS-PVPA are expanded or even partly decomposed, more secondary channels are formed, and the original secondary channels are enlarged, meaning some of the embed catalytic active sites are exposed in the solution of the reaction and the substrates and the reactants can diffuse to these catalytic sites easily through these secondary channels. As the reaction time raise, the conversion rate increases gradually, but the selectivity decreases accordingly. Similar results were obtained in our earlier reports $[13,20]$.

Table 2. The results of the quantity screening of ZPS-PVPA- $\mathrm{SO}_{3} \mathrm{H}$ in the epoxidation of soybean oil. [a]

\begin{tabular}{|c|c|c|c|c|c|}
\hline Entry & ZPS-PVPA-SO ${ }_{3} \mathrm{H}(w t \%)(h)$ & Temperature $\left({ }^{\circ} \mathrm{C}\right)$ & Conversion $(\%)^{[b]}$ & Selectivity $(\%)^{[b]}$ & Yield (\%) \\
\hline 1 & $1(6)$ & 80 & 32.1 & 50.7 & 16.27 \\
\hline 2 & $2(6)$ & 80 & 53.7 & 57.2 & 30.72 \\
\hline 3 & $5(6)$ & 80 & 69.1 & 61.6 & 42.57 \\
\hline 4 & $10(6)$ & 80 & 78.2 & 76.8 & 60.06 \\
\hline 5 & $20(6)$ & 80 & 81.6 & 73.2 & 59.73 \\
\hline 6 & No catalyst (6) & 80 & 10.6 & 3.2 & 0.34 \\
\hline 7 & $10(6)$ & 40 & 36.8 & 43.7 & 16.08 \\
\hline 8 & $10(6)$ & 100 & 81.6 & 53.7 & 43.82 \\
\hline 9 & $10(2)$ & 80 & 47.3 & 56.2 & 26.58 \\
\hline 10 & $10(4)$ & 80 & 59.7 & 63.7 & 38.03 \\
\hline 11 & $10(10)$ & 80 & 83.1 & 57.0 & 47.37 \\
\hline
\end{tabular}

Note: ${ }^{\text {[a] }}$ Reactions were carried out at $80^{\circ} \mathrm{C}$ in 1,2-dichloroethane $(5 \mathrm{~mL})$ with soybean oil $(10 \mathrm{~g})$, TBHP $(5.0 \mathrm{mmol})$, and the ZPS-PVPA-SO3H catalyst $(10 \mathrm{~mol} \%(\mathrm{wt} \%)) ;{ }^{[b]}$ conversions and selectivity were determined by GB/T1676-008 and GB/T 1677-2008 method.

In order to determine the optimal amount of ZPS-PVPA- $\mathrm{SO}_{3} \mathrm{H}$, the reaction was firstly carried out in the presence of different amounts $\left(1-20 \mathrm{wt} \%\right.$, based on soybean oil) at $80^{\circ} \mathrm{C}$ in 1,2-dichloroethane 
using TBHP as an oxidant. The results showed that the conversions were increased from 32.1 to $81.3 \%$ with an increase in yield from 16.27 to $60.06 \%$ when the amount of catalyst was increased from 1 to $20 \mathrm{wt} \%$. Moreover, an additional increase in the amount of catalyst did not show any positive effect on the conversion and selectivity of the soybean oil epoxide (Table 2, entry 5). Blank experiment showed that the ZPS-PVPA- $\mathrm{SO}_{3} \mathrm{H}$ alone is inactive towards epoxidation of soybean oil, and a yield of only $0.34 \%$ was achieved (Table 2, entry 6 ). In addition, we screened the effects of different temperatures on the epoxidation of soybean oil. The results show that the conversion and selectivity increases with increasing temperature but the selectivity decreases when the temperature increases above $80^{\circ} \mathrm{C}$. A similar trend was found for the effect of reaction time on catalytic reactions. This is mainly attributed to the slow decomposition of TBHP, making formation of a by-product more likely at higher temperatures and over longer durations. Therefore, $80^{\circ} \mathrm{C}$ and $6 \mathrm{~h}$ were chosen as the reaction time and temperature of the system.

\subsection{Reusability of the $\mathrm{ZPS}-\mathrm{PVPA}-\mathrm{SO}_{3} \mathrm{H}$}

To assess the recyclability of the ZPS-PVPA- $\mathrm{SO}_{3} \mathrm{H}$, the reaction system was separated by centrifugation after the reaction is completed, the catalyst was left untreated, and the reaction was continuously reacted according to the initial ratio. As shown in Table 3, it is obvious that the catalyst worked well for up to five cycles with no considerable decrease in reactivity (yield\% from 60.06 to $55.25 \%$ ). An unexpected discovery was that the catalytic activity of the catalyst was gradually restored at the 8 th to 10 th cycles of the catalyst when the ZPS-PVPA- $\mathrm{SO}_{3} \mathrm{H}$ was allowed to stand in $2 \mathrm{~mol} / \mathrm{L}$ of dilute hydrochloric acid overnight after the 7 th reuse; similar results have been reported in the asymmetric epoxidation of olefin catalyzed by ZPS-PVPA-SalenMn [12]. This novel phenomenon is probably due to the nano-layered self-supporting function [21] of the inorganic zirconium phosphate portion of the catalyst structure. The catalyst may have a certain "memory function" and can be roughly restored to the original morphology in acid medium. However, the specific reasons remain to be further studied.

Table 3. The recycling of ZPS-PVPA-SO $3 \mathrm{H}$ in epoxidation of soybean oil. ${ }^{[a]}$

\begin{tabular}{cccc}
\hline Run & ${\text { Conversion }(\mathbf{\%})^{[\mathbf{b}]} \mathbf{( h )}}$ Selectivity $(\mathbf{\%})^{[\mathbf{b}]}$ & Yield (\%) \\
\hline 1 & $78.2(6)$ & 76.8 & 60.06 \\
2 & $77.9(6)$ & 75.4 & 58.74 \\
3 & $77.1(6)$ & 74.2 & 57.21 \\
4 & $76.8(6)$ & 73.8 & 56.68 \\
5 & $76.0(6)$ & 72.7 & 55.25 \\
6 & $70.1(6)$ & 66.3 & 46.48 \\
7 & $61.3(6)$ & 50.9 & 31.20 \\
8 & $76.3(6)$ & 69.4 & 52.95 \\
9 & $75.8(6)$ & 67.8 & 51.39 \\
10 & $70.6(6)$ & 62.9 & 44.41 \\
\hline
\end{tabular}

Note: ${ }^{[a]}$ Reactions were carried out at $80^{\circ} \mathrm{C}$ in 1,2 -dichloroethane $(5 \mathrm{~mL})$ with soybean oil $(10 \mathrm{~g}), \mathrm{TBHP}(5.0 \mathrm{mmol})$, and the ZPS-PVPA-SO3H catalyst $(10 \mathrm{~mol} \%(\mathrm{wt} \%)) ;{ }^{\mathrm{b}]}$ conversions and selectivity were determined by GB/T1676-2008 and GB/T 1677-2008 method.

\section{Material and Methods}

\subsection{Materials}

Zirconyl chloride octahydrate, $\gamma$-propyl mercaptotrimethoxysilane (MPTMS), tert-butyl hydroperoxide (TBHP, 65\%, wt \%), and benzoyl peroxide (BPO) were supplied by Alfa Aesar (Tianjian, China). The 1-phenylvinyl phosphonic acid (PVPA) was prepared according to literature [22] and its structures were confirmed by ${ }^{1} \mathrm{H}$ NMR, ${ }^{31} \mathrm{P}$ NMR, and FT-IR. ${ }^{1} \mathrm{H}$ NMR $\left(\mathrm{CDCl}_{3}\right): 6.06(\mathrm{~d}, 1 \mathrm{H}), 6.23$ (d, 1H), 7.26-7.33 (m, 3H), 7.48 (m, 2H). ${ }^{31} \mathrm{P}$ NMR (CD $\left.\mathrm{CD}_{3} \mathrm{OD}\right): 15.9 . \mathrm{IR}(\mathrm{KBr}): 2710,2240,1500,1200,1040$, 
$950,780,720,700 \mathrm{~cm}^{-1}$. Other commercially available chemicals were laboratory-grade reagents from local suppliers. Soybean oil was purchased from a local supermarket ([I.N. $\left.]_{\mathrm{i}}=120\right)$.

\subsection{Methods}

XPS were recorded on ESCALab250 instrument (Thermo Fisher Scientific, USA). SEM were performed on SU8010 (JEOL, Japan) microscopy. EDS were performed on a JSM-7800F (JEOL, Japan) apparatus. TG-DSC analyses were performed on a SBTQ600 Thermal Analyzer (USA) with a heating rate of $20^{\circ} \mathrm{C} \cdot \mathrm{min}^{-1}$ from 25 to $1000{ }^{\circ} \mathrm{C}$ under flowing $\mathrm{N}_{2}\left(100 \mathrm{~mL} \cdot \mathrm{min}^{-1}\right)$. The interlayer spacings were recorded on a LabXRD-6100 automated X-ray power diffractometer using $\mathrm{Cu}-\mathrm{K} \alpha$ radiation and internal silicon powder standard for all samples (Shimadz, Japan). The patterns were generally measured between 2.00 and $80.00^{\circ}$ and $X$-ray tube settings of $40 \mathrm{kV}$ and $5 \mathrm{~mA}$.

\subsection{Preparation of Catalysts}

\subsubsection{Synthesis of Zirconium poly(styrene-phenylvinyl-phosphonate)-phosphate (ZPS-PVPA)}

The synthesis and characterization of ZPS-PVPA (Scheme 1) has been reported earlier by our group [22].

\section{(ZPS-PVPA)}

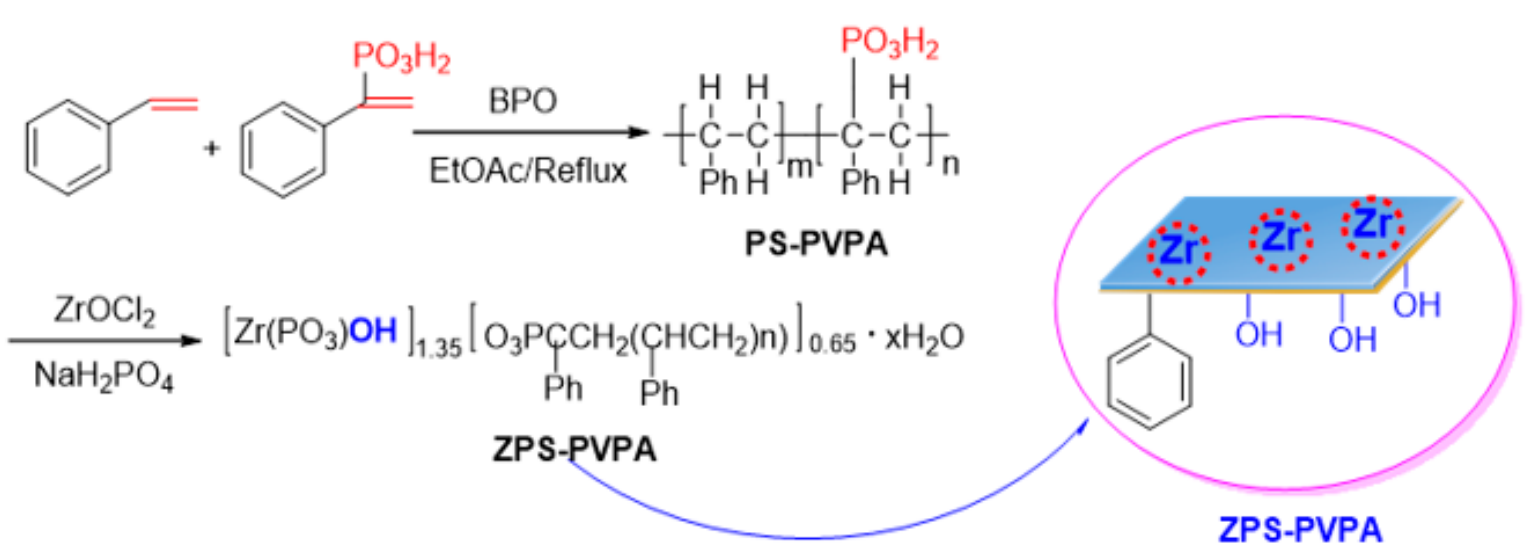

Scheme 1. Synthesis of ZPS-PVPA.

3.3.2. Synthesis of Sulfonic Acid-Functionalized Zirconium Poly(styrene-phenylvinyl-phosphonate)-phosphate

ZPS-PVPA was functionalized by the condensation reaction between the -OH on the ZPS-PVPA surface and the $-\mathrm{Si}(\mathrm{OMe})_{3}$ groups of MPTMS [23]. Here, $0.50 \mathrm{~g}$ of (ZPS-PVPA) was swelled and ultrasounded in toluene $(30 \mathrm{~mL})$ for an hour. Subsequently, MPTMS $(2 \mathrm{~mL})$ was dropped into the suspension and then refluxed under vigorous stirring for $24 \mathrm{~h}$ in a $\mathrm{N}_{2}$ atmosphere. After the reaction, the mixture was filtered and the obtained solid was washed with toluene $(30 \mathrm{~mL} \times 3)$ to remove the residual MPTMS and dried at $60{ }^{\circ} \mathrm{C}$ overnight in a vacuum oven. The obtained product was abbreviated as ZPS-PVPA-SH. ZPS-PVPA-SO ${ }_{3} \mathrm{H}$ was obtained as follows: a certain amount of $\mathrm{H}_{2} \mathrm{O}_{2}$ $(5.5 \mathrm{~mL})$ was slowly added to the dispersion of ZPS-PVPA-SH $(0.3 \mathrm{~g})$ in $12.0 \mathrm{~mL}$ of methanol. The reaction was kept at room temperature for $24 \mathrm{~h}$. The obtained solid was further treated with $\mathrm{HCl}$ $(2.5 \mathrm{~mL}, 37 \mathrm{wt} \%)$ at ambient temperature for complete protonation [24]. The sample was separated by centrifugation and then washed with water and ethanol. After drying at $60^{\circ} \mathrm{C}$ for $24 \mathrm{~h}$ in a vacuum oven, ZPS-PVPA-SO ${ }_{3} \mathrm{H}$ was obtained (Scheme 2). 


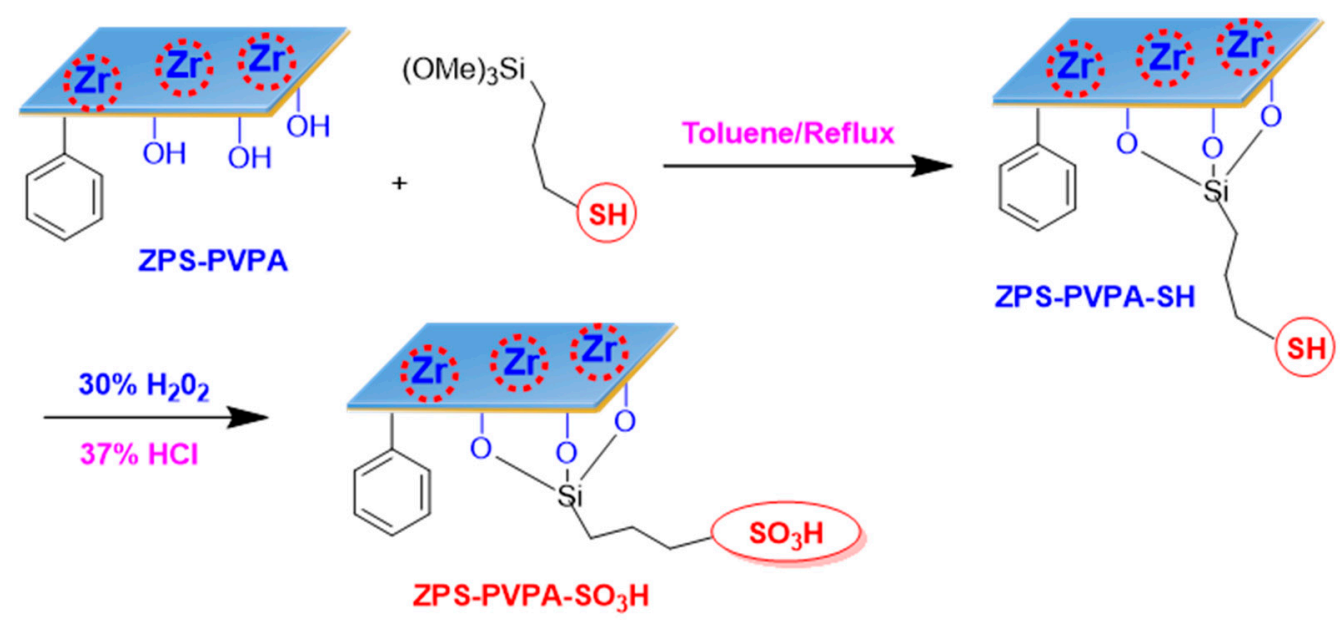

Scheme 2. Synthesis of sulfonic acid-functionalized zirconium poly(styrene-phenylvinyl-phosphonate)phosphate (ZPS-PVPA).

\subsubsection{Epoxidation of Soybean Oil}

The epoxidation of soybean oil in the present of ZPS-PVPA- $\mathrm{SO}_{3} \mathrm{H}$ was performed as follows. A solution of $10 \mathrm{~g}$ soybean oil (about $50.43 \mathrm{mmol}$ in double bonds) and ZPS-PVPA-SO $\mathrm{H}_{3} \mathrm{H}(10 \%$, wt $\%)$ in 1,2-dichloroethane $(10 \mathrm{~mL})$ was mixed at desired temperatures. Then, TBHP (5.43 mmol) was slowly added over $10 \mathrm{~min}$. After the completion of the reaction, the catalyst was centrifuged and the filtrate was washed with hot water until neutral and dried. The evolution over time of the epoxidation reaction, in terms of double bonds conversion and yield and selectivity to epoxide, was followed by determining both O.N. (expressed as grams of epoxy oxygen per $100 \mathrm{~g}$ of oil) and I.N. (expressed as grams of $\mathrm{I}_{2}$ per $100 \mathrm{~g}$ of oil). I.N. was determined through the GB/T1676-2008 method, and O.N. was determined through the GB/T1677-2008 method. The conversion and the yield to epoxide were calculated by the following equations:

$$
\begin{gathered}
\text { Conversion }(\%)=[\text { I.N. }]_{\mathrm{I}}-[\mathrm{I} . \mathrm{N} .]_{\mathrm{f}} /[\mathrm{I} . \mathrm{N} .]_{\mathrm{i}} \times 100 \\
\text { Selectivity }(\%)=\left[\left([\mathrm{O} . \mathrm{N} .]_{\mathrm{f}} / \mathrm{M} . \mathrm{W}(\mathrm{O}) \times 100\right)\right] /\left[[\mathrm{I} . \mathrm{N} .]_{\mathrm{i}}-[\mathrm{I} . \mathrm{N} .]_{\mathrm{f}} /\left(\mathrm{M} . \mathrm{W}\left(\mathrm{I}_{2}\right) \times 100\right)\right] \times 100 \\
\text { Yield to epoxide }(\%)=\text { Conversion } \times \text { Selectivity } \times 100
\end{gathered}
$$

where $i$ and $\mathrm{f}$ represent, respectively, initial and final values, M.W is the molecular weight, O.N. is epoxy value, I.N. is iodine value, $\mathrm{M}(\mathrm{O})=16, \mathrm{M}\left(\mathrm{I}_{2}\right)=254$.

\section{Conclusions}

This paper provides a new solid acid catalyst for catalyzing epoxidized soybean oil. The catalytic results showed that the ZPS-PVPA- $\mathrm{SO}_{3} \mathrm{H}$ can efficiently promote the epoxidation of soybean oil. Furthermore, the ZPS-PVPA- $\mathrm{SO}_{3} \mathrm{H}$ could be easily separated from the products and recycled at least five times without significant loss of catalytic activity. Interestingly, the ZPS-PVPA- $\mathrm{SO}_{3} \mathrm{H}$ was kept in $2 \mathrm{~mol} / \mathrm{L}$ of $\mathrm{HCl}$ overnight at the end of the 7th reaction, and the catalytic activity was gradually restored during the 8 th to10th cycles.

Author Contributions: X.C.Z. designed the experiments and wrote the paper. X.C.Z., X.Y.N., Z.W.T., Y.W., and C.W. performed the experiments. K.Y.S. analyzed the data and provided meaningful advice. X.Z. secured the funds.

Funding: This research was funded by the Program for Innovation Team Building at Institutions of Higher Education in Chongqing (CXTDX201601040), Natural Science Foundation of Chongqing (No. cstc 2018jcyjAX0110). 
Acknowledgments: The authors acknowledge the Science and Technology Research Program of Chongqing Municipal Education Commission (No. KJ201801607, KJQN201801602), and the Key Laboratory for Green Chemical Technology of Chongqing University of Education (No. 2016xjpt08).

Conflicts of Interest: The authors declare no conflict of interest.

\section{References}

1. Goud, V.V.; Patwardhan, A.V.; Dinda, S.; Pradhan, N. Kinetics of epoxidation of jatropha oil with peroxyacetic and peroxyformic acid catalysed by acidic ion exchange resin. Chem. Eng. Sci. 2007, 62, 4065-4076. [CrossRef]

2. Zhang, S.Y.; Jiang, P.P.; Leng, Y.; Xu, Y.C.; Mo, G.T.; Bian, G. Synthesis of cis-dioxomolybdenum(VI)-tridentate schiff base complexes and its catalytic activity on the epoxidation of soybean oil. Chem. J. Chin. Univ. 2013, 34, 1703-1708.

3. Turco, R.; Pischetola, C.; Di Serio, M.; Vitiello, R.; Tesser, R.; Santacesaria, E. Selective Epoxidation of Soybean Oil in the Presence of H-Y Zeolite. Ind. Eng. Chem. Res. 2017, 56, 7930-7936. [CrossRef]

4. Vianello, C.; Piccolo, D.; Lorenzetti, A.; Salzano, E.; Maschio, G. Study of soybean oil epoxidation: Effects of sulfuric acid and the mixing program. Ind. Eng. Chem. Res. 2018, 57, 11517-11525. [CrossRef]

5. Varyambath, A.; Kim, M.R.; Kim, I. Sulfonic acid-functionalized organic knitted porous polyaromatic microspheres as heterogeneous catalysts for biodiesel production. New J. Chem. 2018, 42, 12745-12753. [CrossRef]

6. Chang, J.M.; Guan, X.Y.; Pan, S.Y.; Jia, M.L.; Chen, Y.; Fan, H.J. Sulfonated poly(styrene-divinylbenzene-glycidyl methacrylate)-capsulated magnetite nanoparticles as a recyclable catalyst for one-step biodiesel production from high free fatty acid-containing feedstocks. New J. Chem. 2018, 42, 13074-13080. [CrossRef]

7. Cano, S.E.; Campos, M.J.M.; Fierro, J.L.G. Sulfonic acid-functionalized silica through quantitative oxidation of thiol groups. Chem. Commun. 2003, 2, 246-247. [CrossRef]

8. Rhee, C.H.; Kim, H.K.; Chang, H.; Lee, J.S. Nafion/sulfonated montmorillonite composite: A new concept electrolyte membrane for direct methanol fuel cells. Chem. Mater. 2005, 17, 1691-1697. [CrossRef]

9. Smuleac, V.; Butterfield, D.A.; Sikdar, S.K.; Varma, R.S.; Bhattacharyya, D. Polythiol-functionalized alumina membranes for mercury capture. J. Membr. Sci. 2005, 251, 169-178. [CrossRef]

10. Felice, V.; Ntais, S.; Tavares, A.C. Propyl sulfonic acid functionalization of faujasite-type zeolites: Effect on water and methanol sorption and on proton conductivity. Microporous Mesoporous Mater. 2013, 169, 128-136. [CrossRef]

11. Zhou, Y.J.; Huang, R.C.; Ding, F.C.; Brittain, A.D.; Liu, J.J.; Zhang, M.; Xiao, M.; Meng, Y.Z.; Sun, L.Y. Sulfonic acid-functionalized $\alpha$-zirconium phosphate single-layer nanosheets as a strong solid acid for heterogeneous catalysis applications. ACS Appl. Mater. Interfaces 2014, 6, 7417-7425. [CrossRef] [PubMed]

12. Zou, X.C.; Wang, Y.; Wang, C.; Shi, K.Y.; Ren, Y.R.; Zhao, X. Chiral MnIII (Salen) immobilized on organic polymer/inorganic zirconium hydrogen phosphate functionalized with 3-aminopropyltrimethoxysilane as an efficient and recyclable catalyst for enantioselective epoxidation of styrene. Polymers 2019, 11, 212. [CrossRef] [PubMed]

13. Zou, X.C.; Wang, C.; Wang, Y.; Shi, K.Y.; Wang, Z.M.; Li, D.W.; Fu, X.K. Chiral MnIII (Salen) covalently bonded on modified ZPS-PVPA and ZPS-IPPA as efficient catalysts for enantioselective epoxidation of unfunctionalized olefins. Polymers 2017, 9, 108. [CrossRef]

14. Zou, X.C.; Shi, K.Y.; Li, J.; Wang, Y.; Wang, C.; Deng, C.F.; Ren, Y.R.; Tan, J.; Fu, X.K. Research progress on epoxidation of olefins catalyzed by $\mathrm{Mn}(\mathrm{II}, \mathrm{III}, \mathrm{V})$ in different valence states. Chin. J. Org. Chem. 2016, 36, 1765-1778. [CrossRef]

15. Luo, Y.F.; Zou, X.C.; Fu, X.K.; Huang, X.M.; Jia, Z.Y. The advance in asymmetric epoxidation of olefins catalyzed by chiral Mn (salen). Sci. China Chem. 2011, 41, 433-450.

16. Zou, X.C.; Huang, L.Y.; Quan, W.X.; Wang, C.; Wang, Y.; Zhao, X.; Tan, Z.W. Sulfonic-functionalized organic polystyrene/inorganic hydrogen zirconium phosphate catalyzed epoxidation of soybean oil. Chin. J. Inorg. Chem. 2019, 35, 1349-1356.

17. Alberti, G.; Casciola, M.; Palombari, R. Acid zirconium phosphates and phosphonates as proton conductors and their use for solid-state gas sensors. Russ. J. Electrochem. 1993, 29, 1257-1264. 
18. Sun, S.Q.; Yang, F.C.; Yi, Y.J.; Chang, Y.; Zhou, Y.S.; Zha, F. Catalytic synthesis of epoxidized soybean oil by tridentate pyridine schif-base molybdenum (VI) complex. Fine Chem. 2016, 33, 792-796.

19. Wu, F.J.; Jiang, P.P.; Zhang, P.B.; Dong, Y.M.; Li, X.L. Cu-salen complex as a catalyst for epoxidation of soybean oil to synthesize an environmental friendly plasticizer. China Oils Fats 2012, 37, 56-60.

20. Ren, W.S.; Fu, X.K.; Bao, H.B.; Bai, R.F.; Ding, P.P.; Sui, B.L. Enantioselective epoxidation of unfunctionalized olefins catalyzed by chiral salen $\mathrm{Mn}(\mathrm{III})$ catalyst immobilized on zirconium oligostyrenylphosphonate-phosphate. Catal. Commun. 2009, 10, 788-793. [CrossRef]

21. Wu, X.J.; Ma, X.B.; Ji, Y.L.; Wang, Q.; Jia, X.; Fu, X.K. Synthesis and characterization of a novel type of self-assembled chiral zirconium phosphonates and its application for heterogeneous asymmetric catalysis. J. Mol. Catal. A Chem. 2007, 265, 316-322. [CrossRef]

22. Zou, X.C.; Shi, K.Y.; Wang, C. Chiral MnIII (Salen) supported on tunable phenoxyl group modified zirconium poly(styrene-phenylvinylphosphonate)-phosphate as an efficient catalyst for epoxidation of unfunctionalized olefins. Chin. J. Catal. 2014, 35, 1446-1455. [CrossRef]

23. Park, J.W.; Park, Y.J.; Jun, C.H. Post-grafting of silica surfaces with pre-functionalized organosilanes: New synthetic equivalents of conventional trialkoxysilanes. Chem. Commun. 2011, 47, 4860-4871. [CrossRef]

24. Alberti, G.; Casciola, M.; Costantino, U. Inorganic ion-exchange pellicles obtained by delamination of alpha-zirconium phosphate crystals. J. Colloid Interface Sci. 1985, 107, 256-263. [CrossRef]

(C) 2019 by the authors. Licensee MDPI, Basel, Switzerland. This article is an open access article distributed under the terms and conditions of the Creative Commons Attribution (CC BY) license (http://creativecommons.org/licenses/by/4.0/). 